\title{
Feature and Dissimilarity Representations for the Sound-Based Recognition of Bird Species
}

\author{
José Francisco Ruiz-Muñoz ${ }^{1}$, Mauricio Orozco-Alzate ${ }^{1,2, \star}$, \\ and César Germán Castellanos-Domínguez ${ }^{1}$
}

1 Signal Processing and Recognition Group, Universidad Nacional de Colombia Sede Manizales, $\mathrm{km} 7$ vía al aeropuerto, Manizales (Caldas), Colombia

\{jfruizmu, morozcoa, cgcastellanosd\}@unal.edu.co

2 Departamento de Informática y Computación, Universidad Nacional de Colombia Sede Manizales, km 7 vía al aeropuerto, Manizales (Caldas), Colombia

\begin{abstract}
Pattern recognition and digital signal processing techniques allow the design of automated systems for avian monitoring. They are a non-intrusive and cost-effective way to perform surveys of bird populations and assessments of biological diversity. In this study, a number of representation approaches for bird sounds are compared; namely, feature and dissimilarity representations. In order to take into account the nonstationary nature of the audio signals and to build robust dissimilarity representations, the application of the Earth Mover's Distance (EMD) to time-varying measurements is proposed. Measures of the leave-one-out 1-NN performance are used as comparison criteria. Results show that, overall, the Mel-ceptrum coefficients are the best alternative; specially when computed by frames and used in combination with EMD to generate dissimilarity representations.
\end{abstract}

Keywords: Automated avian monitoring, bird sounds, dissimilarity representations, feature representations.

\section{Introduction}

Advances in pattern recognition and digital signal processing allow the identification of bird species by their emitted sounds and, thereby, the design of automated systems for avian monitoring. In spite of those advances, biodiversity assessments have typically been carried out by visual inspection, which requires human involvement and, therefore, may be expensive and have a limited coverage. In contrast, automatic acoustic monitoring is a non-intrusive and costeffective alternative that may provide good temporal and spatial coverages.

The simplest sounds in a bird song are called elements or notes. Several notes together in a regular pattern in a song constitute a syllable and, in turn, several syllables are a song phrase [1]. Previous studies [2 4] have shown that the sound-based recognition of bird species is suitable when considering syllables as

\footnotetext{
* Mauricio Orozco-Alzate is a member of Sociedad Caldense de Ornitología (SCO), a regional ornithological society from Caldas, Colombia: http://rnoa.org/sco/ 
elementary units. Raw measurements corresponding to those elementary units have to be represented in vector spaces where classification rules can afterwards be applied. Representations for bioacoustic signals have traditionally been built by feature extraction; however, we advocate that dissimilarity representations are also a feasible option to face this problem. Furthermore, dissimilarities have the potential to build either simpler or richer representations; the later case, a richer representation, when considering for instance time-varying measurements that take into account non-stationarity. In this study, we evaluate different types of representations, including feature-based and dissimilarity-based ones, for bird sounds segmented into syllables.

Considered feature representations include the so-called standard features and a so-called coarse representation of segment structure; both of them include the syllable duration as well as features related to particular frequencies and maximum values in the frequency domain [2]. Besides, we evaluate a set of acoustical features, named in [3] and here as descriptive features, and the Mel-cepstrum representation, which is based on a linear cosine transform of a log power spectrum on a nonlinear Mel scale of frequency. Spectral analyses such as as the Fast Fourier Transform (FFT) and the Parametric estimation of the Power spectral Density (PSD) are simple initial representations to find dissimilarities between syllables. Such options to build dissimilarity representations are also considered. In addition, we calculate dissimilarities with richer spectral estimates, namely time-varying ones, that consist in dividing segments into frames and mapping each one into two-dimensional spectral or feature representations. The computation of dissimilarities between time-varying initial representations is carried out by using the Earth Mover's Distance (EMD), due to its usefulness to compare distributions.

The goodness of a particular representation can be roughly assessed by using measures of the leave-one-out nearest-neighbor (1-NN) performance. Such measures are commonly used as criteria when selecting features or prototypes for a representation [5]. We use them here as comparison values between the evaluated representations.

\section{Methods}

The design of a bird sound recognition system includes, at least, the following three stages: preprocessing, representation and performance evaluation. The first one consists in the segmentation of continuous records, whose objective is to detect intervals - according to the energy signal - where there are sounds emitted by birds. Consequently, it is assumed that bird sounds are located in signal regions with high energy levels. Steps of the segmentation stage are: computation of the energy signal, estimation of an energy threshold, search of syllables (regions having energies above a threshold), and the application of a criterion of deletion and merging of very short segments. 
Regarding the second stage - representation - several methods of featurebased representations, commonly used in bioacoustics and bird sound classification, are compared in this paper. In addition, the dissimilarity-based approach is proposed as an alternative for representation. The last stage - performance evaluation - is carried out, as indicated at the end of Sec. 1] i.e. by using the leave-one-out 1-NN performance.

\subsection{Feature Representations}

Standard features: Segments are characterized by using four features as proposed in [2]; namely minimum and maximum frequencies, temporal duration and maximum power.

Coarse representation of segment structure: The following eleven variables, originally proposed in [2], are used as features for each segment: minimum and maximum frequencies, temporal duration and frequency of maximum power in eight non-overlapping frames.

Descriptive features: This set includes both temporal and spectral features. Segments are divided into overlapping frames of 256 samples with $50 \%$ overlap. For each frame, the following features are estimated: spectral centroid, signal bandwidth, spectral roll-off frequency, spectral flux, spectral flatness, zero crossing rate and short time energy. Feature vectors for classification are composed by mean and variance values of the feature trajectories along the frames. Frequency range (minimum and maximum frequencies), segment temporal duration and modulation spectrum (position and magnitude of the maximum peak in the modulation spectrum) are calculated from the entire segment. Therefore, 19 features are calculated with this method as proposed in [3].

Mel-cepstrum representation: Mel-frequency cepstral coefficients (MFCCs) are a feature representation method commonly used in many audio classification problems, e.g. in speech recognition. Mel-frequency scale is derived from the human perceptual system. Such systems in birds are not the same but exhibit similar characteristics; therefore, MFCCs have also been used in birdsong recognition [3, 4]. The first $12 \mathrm{MFCCs,} \mathrm{the} \mathrm{log-energy} \mathrm{and} \mathrm{the} \mathrm{so-called} \mathrm{delta}$ and delta-delta coefficients are obtained for each frame. Their mean values along the frames are used as features, as proposed in [3].

\subsection{Dissimilarity Representations}

A dissimilarity representation consists in building vectorial spaces where coordinate axes represent dissimilarities - typically distance measures - to prototypes. In these spaces, classifiers can be built. In a full dissimilarity matrix, prototypes are all the elements available in a particular dataset. The matrix is often symmetric and must be real and have zero diagonal. "Dissimilarity representations can 
be derived in many ways, e.g. from raw (sensor) measurements such as images, histograms or spectra or, from an initial representation by features, strings or graphs" [5]. Considering that the analysis of signal properties is usually done in the frequency domain, we have calculated the spectrum for each signal by using two different approaches: FFT and PSD. Dissimilarity representations have been then computed by pointwise distances between spectra.

Dissimilarity representations, derived as described above, suppose that spectral behavior is similar in the entire segment. In order to obviate such an assumption, we also use representations that change over time (time-varying). In such a way, the acoustic space for each sound segment is efficiently covered [6]. Time-varying representations are computed by dividing sound segments into frames and converting each one to either a spectral or a feature representation. Feature sets measured for each frame were: 1) spectrogram, also known as short time Fourier transform (SFT); 2) PSD by using the Yule Walker method; 3) selected descriptive features (spectral centroid, signal bandwidth, spectral rolloff frequency, spectral flux, spectral flatness, zero crossing rate and short time energy); and 4) the Mel-cepstrum representation as explained in Sec. 2.1. Sets 3 ) and 4) must be standardized because features are not in same scale.

Measuring dissimilarities between representations: In the case of equallysized representations (e.g. FFT or PSD) a classical measure, as the Euclidean distance, can be used. Conversely, the Euclidean distance can not be directly applied to time-varying representations. To overcome this difficulty, in this study we have used the EMD. Due to space constraints, we are not able to provide a description for this distance measure; see [7] and [6] for further implementation details.

\section{Experimental Results}

We performed a set of experiments on a dataset of raw field recordings taken at Reserva Natural Río Blanco in Manizales, Colombia. The sampling frequency of the recordings is $44.1 \mathrm{kHz}$. The dataset is composed by a total of 595 syllables distributed per species as follow: 1: Grallaria ruficapilla (GR, 33), Henicorhina leucophrys (HL, 64), Mimus gilvus (MG, 66), Myadestes ralloides (MR, 58), Pitangus sulphuratus (PS, 53), Pyrrhomyias cinnamomea (PC, 36), Troglodytes aedon (TA, 33), Turdus ignobilis (TI, 74), Turdus serranus (TS, 78), Xiphocolaptes promeropirhynchus (XP, 46) and Zonotrichia capensis (ZC, 54).

Evaluation for each representation was assessed by using measures of the leave-one-out 1-NN performance. For each representation, a confusion matrix is reported. In addition, the following performance measures per class are presented: True Positive rate (TP), False Positive rate (FP), Accuracy (ACC) and F1 score. Results are shown in Tables 1, 4 .

${ }^{1}$ Scientific names are indicated together with a pair (Abbreviation, Number of syllables). 
Table 1. Results for feature representations

(a) Standard features

\begin{tabular}{|c|c|c|c|c|c|c|c|c|c|c|c|c|c|c|c|c|}
\hline & \multicolumn{11}{|c|}{ Predicted } & \multirow[b]{2}{*}{ - Total } & \multirow{2}{*}{ TP } & \multirow{2}{*}{ FP } & \multirow{2}{*}{$\mathrm{ACC}$} & \multirow{2}{*}{ F1 } \\
\hline & GR & $\mathrm{HL}$ & MG & $\overline{M R}$ & PS & PC & TA & TI & TS & $\mathrm{XP}$ & $\overline{Z C}$ & & & & & \\
\hline GR & 21 & 0 & 0 & 3 & 1 & 3 & 0 & 0 & 1 & 4 & 0 & 33 & 63.64 & 1.60 & 96.47 & 66.67 \\
\hline $\mathrm{HL}$ & 1 & 35 & 8 & 2 & 4 & 0 & 7 & 1 & 2 & 3 & 1 & 64 & 54.69 & 4.33 & 91.26 & 57.38 \\
\hline MG & 0 & 6 & 28 & 4 & 10 & 4 & 5 & 2 & 3 & 1 & 3 & 66 & 42.42 & 5.67 & 88.57 & 45.16 \\
\hline MR & 1 & 1 & 3 & 39 & 3 & 5 & 0 & 3 & 3 & 0 & 0 & 58 & 67.24 & 2.98 & 94.12 & 69.03 \\
\hline ख PS & 0 & 2 & 4 & 2 & 36 & 1 & 2 & 1 & 3 & 2 & 0 & 53 & 67.92 & 4.98 & 92.61 & 62.07 \\
\hline $\mathrm{PC}$ & 2 & 1 & 4 & 2 & 0 & 24 & 0 & 0 & 0 & 3 & 0 & 36 & 66.67 & 2.50 & 95.63 & 64.86 \\
\hline$\& \mathrm{TA}$ & 0 & 6 & 3 & 0 & 2 & 0 & 21 & 0 & 0 & 0 & 1 & 33 & 63.64 & 3.02 & 95.13 & 59.15 \\
\hline TI & 0 & 0 & 1 & 1 & 0 & 0 & 1 & 67 & 3 & 1 & 0 & 74 & 90.54 & 2.30 & 96.81 & 87.58 \\
\hline TS & 1 & 5 & 5 & 2 & 4 & 1 & 0 & 4 & 52 & 1 & 3 & 78 & 66.67 & 3.87 & 92.27 & 69.33 \\
\hline $\mathrm{XP}$ & 2 & 1 & 1 & 0 & 1 & 0 & 0 & 0 & 1 & 39 & 1 & 46 & 84.78 & 2.91 & 96.13 & 77.23 \\
\hline $\mathrm{ZC}$ & 2 & 1 & 1 & 0 & 2 & 0 & 2 & 1 & 4 & 1 & 40 & 54 & 74.07 & 1.66 & 96.13 & 77.67 \\
\hline Total & 30 & 58 & 58 & 55 & 63 & 38 & 38 & 79 & 72 & 55 & 49 & 595 & & & & \\
\hline
\end{tabular}

(b) Coarse representation of segment structure

\begin{tabular}{|c|c|c|c|c|c|c|c|c|c|c|c|c|c|c|c|c|}
\hline & \multicolumn{11}{|c|}{ Predicted } & \multirow{2}{*}{ - Total } & \multirow{2}{*}{ TP } & \multirow{2}{*}{ FP } & \multirow{2}{*}{$\mathrm{ACC}$} & \multirow{2}{*}{ F 1} \\
\hline & GR & $\mathrm{HL}$ & MG & $\overline{\mathrm{MR}}$ & PS & $\overline{\mathrm{PC}}$ & TA & TI & TS & $\mathrm{XP}$ & $\overline{\mathrm{ZC}}$ & & & & & \\
\hline GR & 27 & 0 & 3 & 0 & 0 & 0 & 0 & 0 & 3 & 0 & 0 & 33 & 81.82 & 0.71 & 98.32 & 84.38 \\
\hline $\mathrm{HL}$ & 1 & 45 & 2 & 2 & 3 & 0 & 4 & 1 & 2 & 2 & 2 & 64 & 70.31 & 2.07 & 94.96 & 75.00 \\
\hline MG & 0 & 1 & 43 & 2 & 2 & 3 & 1 & 0 & 11 & 2 & 1 & 66 & 65.15 & 4.73 & 91.93 & 64.18 \\
\hline MR & 0 & 0 & 1 & 41 & 1 & 6 & 0 & 1 & 7 & 1 & 0 & 58 & 70.69 & 2.98 & 94.45 & 71.30 \\
\hline ฮี PS & 2 & 0 & 6 & 1 & 40 & 0 & 0 & 0 & 0 & 4 & 0 & 53 & 75.47 & 2.21 & 95.80 & 76.19 \\
\hline $\mathrm{PC}$ & 0 & 0 & 1 & 6 & 0 & 25 & 1 & 0 & 1 & 1 & 1 & 36 & 69.44 & 2.50 & 95.80 & 66.67 \\
\hline$\varangle \mathrm{TA}$ & 0 & 5 & 1 & 0 & 3 & 1 & 22 & 0 & 0 & 0 & 1 & 33 & 66.67 & 1.78 & 96.47 & 67.69 \\
\hline $\mathrm{TI}$ & 0 & 1 & 2 & 1 & 0 & 0 & 0 & 65 & 3 & 2 & 0 & 74 & 87.84 & 0.77 & 97.82 & 90.91 \\
\hline TS & 0 & 2 & 6 & 2 & 0 & 3 & 1 & 2 & 60 & 1 & 1 & 78 & 76.92 & 5.61 & 92.10 & 71.86 \\
\hline $\mathrm{XP}$ & 0 & 0 & 1 & 1 & 1 & 0 & 0 & 0 & 1 & 42 & 0 & 46 & 91.30 & 2.37 & 97.14 & 83.17 \\
\hline $\mathrm{ZC}$ & 1 & 2 & 2 & 1 & 2 & 1 & 3 & 0 & 1 & 0 & 41 & 54 & 75.93 & 1.11 & 96.81 & 81.19 \\
\hline Total & 31 & 56 & 68 & 57 & 52 & 39 & 32 & 69 & 89 & 55 & 47 & 595 & & & & \\
\hline
\end{tabular}

(c) Descriptive features

\begin{tabular}{|c|c|c|c|c|c|c|c|c|c|c|c|c|c|c|c|c|c|}
\hline & \multicolumn{10}{|c|}{ Predicted } & \multirow{2}{*}{\multicolumn{2}{|c|}{$\mathrm{ZC}^{\text {Total }}$}} & \multirow{2}{*}{$\mathrm{TP}$} & \multirow{2}{*}{ FP } & \multirow{2}{*}{$\mathrm{ACC}$} & \multirow{2}{*}{ F1 } \\
\hline & & GR & $\mathrm{HL}$ & MG & $\mathrm{MR}$ & PS & $\mathrm{PC}$ & TA & TI & $\mathrm{TS}$ & $\mathrm{XP}$ & & & & & & \\
\hline & GR & 33 & 0 & 0 & 0 & 0 & 0 & 0 & 0 & 0 & 0 & 0 & 33 & 100.00 & 0.36 & 99.66 & 97.06 \\
\hline & HL & 0 & 54 & 1 & 0 & 0 & 0 & 7 & 1 & 1 & 0 & 0 & 64 & 84.38 & 1.88 & 96.64 & 84.38 \\
\hline & MG & 1 & 2 & 58 & 1 & 3 & 0 & 0 & 0 & 1 & 0 & 0 & 66 & 87.88 & 1.51 & 97.31 & 87.88 \\
\hline & MR & 0 & 0 & 2 & 54 & 0 & 0 & 0 & 1 & 0 & 0 & 1 & 58 & 93.10 & 0.93 & 98.49 & 92.31 \\
\hline J & PS & 0 & 0 & 0 & 0 & 52 & 0 & 0 & 0 & 1 & 0 & 0 & 53 & 98.11 & 0.74 & 99.16 & 95.41 \\
\hline & $\mathrm{PC}$ & 0 & 0 & 0 & 1 & 0 & 34 & 1 & 0 & 0 & 0 & 0 & 36 & 94.44 & 0.00 & 99.66 & 97.14 \\
\hline & TA & 0 & 5 & 2 & 0 & 0 & 0 & 26 & 0 & 0 & 0 & 0 & 33 & 78.79 & 1.42 & 97.48 & 77.61 \\
\hline & TI & 0 & 0 & 1 & 0 & 0 & 0 & 0 & 72 & 1 & 0 & 0 & 74 & 97.30 & 0.38 & 99.33 & 97.30 \\
\hline & TS & 0 & 3 & 1 & 1 & 1 & 0 & 0 & 0 & 70 & 1 & 1 & 78 & 89.74 & 0.77 & 97.98 & 92.11 \\
\hline & $\mathrm{XP}$ & 0 & 0 & 0 & 0 & 0 & 0 & 0 & 0 & 0 & 46 & 0 & 46 & 100.00 & 0.18 & 99.83 & 98.92 \\
\hline & $\mathrm{ZC}$ & 1 & 0 & 1 & 2 & 0 & 0 & 0 & 0 & 0 & 0 & 50 & 54 & 92.59 & 0.37 & 98.99 & 94.34 \\
\hline & Total & 32 & 64 & 65 & 59 & 56 & 34 & 34 & 74 & 74 & 47 & 52 & 595 & & & & \\
\hline
\end{tabular}

(d) Mel-cepstrum representation

\begin{tabular}{|c|c|c|c|c|c|c|c|c|c|c|c|c|c|c|c|c|c|}
\hline & \multicolumn{10}{|c|}{ Predicted } & \multirow{2}{*}{\multicolumn{2}{|c|}{$\mathrm{ZC}^{\text {Total }}$}} & \multirow{2}{*}{$\mathrm{TP}$} & \multirow{2}{*}{ FP } & \multirow{2}{*}{$\mathrm{ACC}$} & \multirow{2}{*}{ F1 } \\
\hline & & GR & $\mathrm{HL}$ & MG & MR & PS & $\mathrm{PC}$ & TA & & TS & $\mathrm{XP}$ & & & & & & \\
\hline & GR & 33 & 0 & 0 & 0 & 0 & 0 & 0 & 0 & 0 & 0 & 0 & 33 & 100.00 & 0.00 & 100.00 & 100.00 \\
\hline & $\mathrm{HL}$ & 0 & 58 & 0 & 0 & 0 & 0 & 5 & 0 & 0 & 1 & 0 & 64 & 90.63 & 1.69 & 97.48 & 88.55 \\
\hline & MG & 0 & 0 & 66 & 0 & 0 & 0 & 0 & 0 & 0 & 0 & 0 & 66 & 100.00 & 0.38 & 99.66 & 98.51 \\
\hline & MR & 0 & 1 & 0 & 54 & 1 & 0 & 1 & 0 & 0 & 0 & 1 & 58 & 93.10 & 0.19 & 99.16 & 95.58 \\
\hline$\vec{\sigma}$ & PS & 0 & 0 & 0 & 0 & 53 & 0 & 0 & 0 & 0 & 0 & 0 & 53 & 100.00 & 0.37 & 99.66 & 98.15 \\
\hline 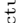 & $\mathrm{PC}$ & 0 & 0 & 0 & 1 & 0 & 34 & 0 & 0 & 1 & 0 & 0 & 36 & 94.44 & 0.18 & 99.50 & 95.77 \\
\hline & TA & 0 & 5 & 0 & 0 & 0 & 0 & 28 & 0 & 0 & 0 & 0 & 33 & 84.85 & 1.25 & 97.98 & 82.35 \\
\hline & TI & 0 & 0 & 1 & 0 & 0 & 0 & 1 & 70 & 0 & 2 & 0 & 74 & 94.59 & 0.38 & 98.99 & 95.89 \\
\hline & TS & 0 & 3 & 1 & 0 & 0 & 1 & 0 & 2 & 69 & 1 & 1 & 78 & 88.46 & 0.58 & 97.98 & 92.00 \\
\hline & XP & 0 & 0 & 0 & 0 & 1 & 0 & 0 & 0 & 2 & 43 & 0 & 46 & 100.00 & 0.18 & 99.83 & 98.92 \\
\hline & $\mathrm{ZC}$ & 0 & 0 & 0 & 0 & 0 & 0 & 0 & 0 & 0 & 0 & 54 & 54 & 100.00 & 0.37 & 99.66 & 98.18 \\
\hline & Total & 33 & 67 & 68 & 55 & 55 & 35 & 35 & 72 & 72 & 47 & 56 & 595 & & & & \\
\hline
\end{tabular}


Table 2. Results for dissimilarity representations computed from 1-D spectra

(a) Spectra computed by FFT

\begin{tabular}{|c|c|c|c|c|c|c|c|c|c|c|c|c|c|c|c|c|}
\hline & \multicolumn{10}{|c|}{ Predicted } & \multirow{2}{*}{\multicolumn{2}{|c|}{$\overline{Z C}^{\text {Total }}$}} & \multirow[b]{2}{*}{$\mathrm{TP}$} & \multirow[b]{2}{*}{ FP } & \multirow[b]{2}{*}{$\mathrm{ACC}$} & \multirow[b]{2}{*}{ F1 } \\
\hline & $\overline{\mathrm{GR}}$ & $\mathrm{HL}$ & MG & $\mathrm{MR}$ & PS & $\mathrm{PC}$ & $\mathrm{TA}$ & TI & TS & $\mathrm{XP}$ & & & & & & \\
\hline GR & 20 & 4 & 1 & 0 & 1 & 0 & 1 & 1 & 1 & 0 & 4 & 33 & 60.61 & 1.42 & 96.47 & 65.57 \\
\hline $\mathrm{HL}$ & 0 & 28 & 6 & 3 & 1 & 4 & 7 & 6 & 3 & 1 & 5 & 64 & 43.75 & 7.91 & 86.89 & 41.79 \\
\hline MG & 1 & 5 & 35 & 1 & 7 & 1 & 0 & 7 & 4 & 4 & 1 & 66 & 53.03 & 7.18 & 88.40 & 50.36 \\
\hline MR & 0 & 2 & 0 & 33 & 2 & 0 & 1 & 6 & 5 & 0 & 9 & 58 & 56.90 & 5.03 & 91.26 & 55.93 \\
\hline ฮี $\mathrm{PS}$ & 1 & 1 & 3 & 5 & 35 & 1 & 0 & 4 & 2 & 0 & 1 & 53 & 66.04 & 3.87 & 93.45 & 64.22 \\
\hline$\exists \mathrm{PC}$ & 1 & 6 & 1 & 1 & 0 & 17 & 1 & 1 & 6 & 0 & 2 & 36 & 47.22 & 3.76 & 93.28 & 45.95 \\
\hline \& TA & 1 & 6 & 4 & 0 & 2 & 1 & 13 & 0 & 1 & 2 & 3 & 33 & 39.39 & 1.78 & 94.96 & 46.43 \\
\hline TI & 1 & 5 & 7 & 3 & 1 & 3 & 0 & 40 & 10 & 3 & 1 & 74 & 54.05 & 7.49 & 87.73 & 52.29 \\
\hline TS & 2 & 5 & 8 & 4 & 5 & 7 & 0 & 10 & 29 & 5 & 3 & 78 & 37.18 & 8.12 & 84.71 & 38.93 \\
\hline $\mathrm{XP}$ & 0 & 5 & 8 & 0 & 1 & 1 & 0 & 2 & 6 & 21 & 2 & 46 & 45.65 & 2.73 & 93.28 & 51.22 \\
\hline $\mathrm{ZC}$ & 1 & 3 & 0 & 10 & 1 & 3 & 0 & 2 & 4 & 0 & 30 & 54 & 55.56 & 5.73 & 90.76 & 52.17 \\
\hline Total & 28 & 70 & 73 & 60 & 56 & 38 & 23 & 79 & 71 & 36 & 61 & 595 & & & & \\
\hline
\end{tabular}

(b) Spectra computed by PSD

\begin{tabular}{|c|c|c|c|c|c|c|c|c|c|c|c|c|c|c|c|c|}
\hline & \multicolumn{10}{|c|}{ Predicted } & \multirow{2}{*}{\multicolumn{2}{|c|}{$\overline{\mathrm{ZC}}^{\text {Total }}$}} & \multirow{2}{*}{ TP } & \multirow{2}{*}{ FP } & \multirow{2}{*}{$\mathrm{ACC}$} & \multirow{2}{*}{$\mathrm{F} 1$} \\
\hline & $\overline{G R}$ & $\mathrm{HL}$ & MG & MR & $\mathrm{PS}$ & $\overline{\mathrm{PC}}$ & TA & TI & TS & $\mathrm{XP}$ & & & & & & \\
\hline GR & 31 & 0 & 1 & 0 & 0 & 0 & 0 & 0 & 0 & 1 & 0 & 33 & 93.94 & 0.18 & 99.50 & 95.38 \\
\hline HL & 0 & 48 & 3 & 1 & 1 & 0 & 5 & 0 & 1 & 1 & 4 & 64 & 75.00 & 3.39 & 94.29 & 73.85 \\
\hline MG & 0 & 4 & 44 & 1 & 2 & 0 & 1 & 3 & 6 & 5 & 0 & 66 & 66.67 & 2.65 & 93.95 & 70.97 \\
\hline $\mathrm{MR}$ & 0 & 1 & 0 & 46 & 1 & 2 & 0 & 2 & 5 & 0 & 1 & 58 & 79.31 & 0.93 & 97.14 & 84.40 \\
\hline$\vec{\pi} \mathrm{PS}$ & 0 & 1 & 0 & 0 & 41 & 2 & 0 & 1 & 3 & 4 & 1 & 53 & 77.36 & 2.03 & 96.13 & 78.10 \\
\hline $\mathrm{PC}$ & 0 & 0 & 0 & 0 & 0 & 34 & 1 & 0 & 1 & 0 & 0 & 36 & 94.44 & 0.89 & 98.82 & 90.67 \\
\hline TA & 0 & 6 & 2 & 1 & 0 & 0 & 23 & 1 & 0 & 0 & 0 & 33 & 69.70 & 1.60 & 96.81 & 70.77 \\
\hline TI & 0 & 0 & 1 & 1 & 1 & 0 & 0 & 71 & 0 & 0 & 0 & 74 & 95.95 & 2.11 & 97.65 & 91.03 \\
\hline TS & 0 & 2 & 2 & 1 & 4 & 1 & 0 & 3 & 58 & 7 & 0 & 78 & 74.36 & 3.29 & 93.78 & 75.82 \\
\hline XP & 1 & 2 & 5 & 0 & 2 & 0 & 1 & 1 & 1 & 33 & 0 & 46 & 71.74 & 3.28 & 94.79 & 68.04 \\
\hline $\mathrm{ZC}$ & 0 & 2 & 0 & 0 & 0 & 0 & 1 & 0 & 0 & 0 & 51 & 54 & 94.44 & 1.11 & 98.49 & 91.89 \\
\hline Total & 32 & 66 & 58 & 51 & 52 & 39 & 32 & 82 & 75 & 51 & 57 & 595 & & & & \\
\hline
\end{tabular}

Table 3. Results for dissimilarity representations derived from:

(a) SFT

\begin{tabular}{|c|c|c|c|c|c|c|c|c|c|c|c|c|c|c|c|c|}
\hline & \multicolumn{11}{|c|}{ Predicted } & \multirow{2}{*}{ Total } & \multirow[b]{2}{*}{$\mathrm{TP}$} & \multirow[b]{2}{*}{ FP } & \multirow[b]{2}{*}{$\mathrm{ACC}$} & \multirow[b]{2}{*}{$\mathrm{F} 1$} \\
\hline & $\overline{\mathrm{GR}}$ & $\mathrm{HL}$ & MG & MR & $\mathrm{PS}$ & $\overline{\mathrm{PC}}$ & $\mathrm{TA}$ & TI & TS & $\mathrm{XP}$ & $\overline{Z C}$ & & & & & \\
\hline GR & 33 & 0 & 0 & 0 & 0 & 0 & 0 & 0 & 0 & 0 & 0 & 33 & 100.00 & 0.00 & 100.00 & 100.00 \\
\hline HL & 0 & 58 & 1 & 0 & 0 & 0 & 5 & 0 & 0 & 0 & 0 & 64 & 90.62 & 1.51 & 97.65 & 89.23 \\
\hline MG & 0 & 2 & 59 & 0 & 0 & 0 & 0 & 1 & 3 & 1 & 0 & 66 & 89.39 & 0.76 & 98.15 & 91.47 \\
\hline MR & 0 & 1 & 0 & 52 & 0 & 0 & 1 & 0 & 4 & 0 & 0 & 58 & 89.66 & 0.00 & 98.99 & 94.55 \\
\hline ฮี PS & 0 & 0 & 0 & 0 & 50 & 0 & 1 & 0 & 1 & 1 & 0 & 53 & 94.34 & 0.18 & 99.33 & 96.15 \\
\hline $\mathrm{PC}$ & 0 & 0 & 0 & 0 & 0 & 36 & 0 & 0 & 0 & 0 & 0 & 36 & 100.00 & 0.18 & 99.83 & 98.63 \\
\hline$\& \mathrm{TA}$ & 0 & 5 & 0 & 0 & 0 & 0 & 26 & 0 & 1 & 1 & 0 & 33 & 78.79 & 1.60 & 97.31 & 76.47 \\
\hline TI & 0 & 0 & 1 & 0 & 0 & 0 & 1 & 72 & 0 & 0 & 0 & 74 & 97.30 & 0.19 & 99.50 & 97.96 \\
\hline TS & 0 & 0 & 0 & 0 & 0 & 1 & 0 & 0 & 76 & 1 & 0 & 78 & 97.44 & 1.74 & 98.15 & 93.25 \\
\hline $\mathrm{XP}$ & 0 & 0 & 2 & 0 & 0 & 0 & 0 & 0 & 0 & 44 & 0 & 46 & 95.65 & 0.73 & 98.99 & 93.62 \\
\hline $\mathrm{ZC}$ & 0 & 0 & 0 & 0 & 1 & 0 & 1 & 0 & 0 & 0 & 52 & 54 & 96.30 & 0.00 & 99.66 & 98.11 \\
\hline Total & 33 & 66 & 63 & 52 & 51 & 37 & 35 & 73 & 85 & 48 & 52 & 595 & & & & \\
\hline
\end{tabular}

(b) Time-varying PSD

\begin{tabular}{|c|c|c|c|c|c|c|c|c|c|c|c|c|c|c|c|c|}
\hline & \multicolumn{11}{|c|}{ Predicted } & \multirow{2}{*}{ Total } & \multirow{2}{*}{$\mathrm{TP}$} & \multirow{2}{*}{ FP } & \multirow{2}{*}{$\mathrm{ACC}$} & \multirow{2}{*}{ F 1} \\
\hline & $\overline{G R}$ & $\mathrm{HL}$ & $\mathrm{MG}$ & $\mathrm{MR}$ & PS & $\mathrm{PC}$ & TA & $\mathrm{TI}$ & $\mathrm{TS}$ & $\mathrm{XP}$ & $\mathrm{ZC}$ & & & & & \\
\hline GR & 33 & 0 & 0 & 0 & 0 & 0 & 0 & 0 & 0 & 0 & 0 & 33 & 100.00 & 0.00 & 100.00 & 100.00 \\
\hline $\mathrm{HL}$ & 0 & 53 & 2 & 0 & 0 & 0 & 7 & 0 & 2 & 0 & 0 & 64 & 82.81 & 3.01 & 95.46 & 79.70 \\
\hline MG & 0 & 1 & 56 & 0 & 0 & 0 & 1 & 0 & 4 & 4 & 0 & 66 & 84.85 & 1.89 & 96.64 & 84.85 \\
\hline MR & 0 & 2 & 0 & 47 & 1 & 0 & 2 & 0 & 5 & 0 & 1 & 58 & 81.03 & 0.37 & 97.82 & 87.85 \\
\hline ๙ี $\mathrm{PS}$ & 0 & 1 & 1 & 0 & 45 & 0 & 0 & 1 & 3 & 2 & 0 & 53 & 84.91 & 0.55 & 98.15 & 89.11 \\
\hline $\mathrm{PC}$ & 0 & 1 & 0 & 0 & 0 & 33 & 1 & 0 & 1 & 0 & 0 & 36 & 91.67 & 0.54 & 98.99 & 91.67 \\
\hline$\stackrel{T}{\mathrm{~T} A}$ & 0 & 5 & 0 & 0 & 0 & 0 & 27 & 0 & 0 & 1 & 0 & 33 & 81.82 & 1.96 & 97.14 & 76.06 \\
\hline TI & 0 & 0 & 2 & 1 & 1 & 1 & 0 & 68 & 1 & 0 & 0 & 74 & 91.89 & 0.77 & 98.32 & 93.15 \\
\hline TS & 0 & 1 & 2 & 1 & 0 & 1 & 0 & 3 & 64 & 6 & 0 & 78 & 82.05 & 3.68 & 94.45 & 79.50 \\
\hline $\mathrm{XP}$ & 0 & 1 & 3 & 0 & 0 & 0 & 0 & 0 & 2 & 40 & 0 & 46 & 86.96 & 2.37 & 96.81 & 80.81 \\
\hline $\mathrm{ZC}$ & 0 & 4 & 0 & 0 & 1 & 1 & 0 & 0 & 1 & 0 & 47 & 54 & 87.04 & 0.18 & 98.66 & 92.16 \\
\hline Total & 33 & 69 & 66 & 49 & 48 & 36 & 38 & 72 & 83 & 53 & 48 & 595 & & & & \\
\hline
\end{tabular}

Total accuracy $=86.22 \%$ 
Table 4. Results for dissimilarity representations derived for frame-based:

(a) Descriptive features

\begin{tabular}{|c|c|c|c|c|c|c|c|c|c|c|c|c|c|c|c|c|}
\hline & \multicolumn{10}{|c|}{ Predicted } & \multirow{2}{*}{\multicolumn{2}{|c|}{$\mathrm{ZC}^{\text {Total }}$}} & \multirow[b]{2}{*}{$\mathrm{TP}$} & \multirow[b]{2}{*}{ FP } & \multirow[b]{2}{*}{$\mathrm{ACC}$} & \multirow[b]{2}{*}{$\mathrm{F} 1$} \\
\hline & GR & $\mathrm{HL}$ & $M G$ & $\overline{M R}$ & PS & $\mathrm{PC}$ & TA & TT & TS & $\mathrm{XP}$ & & & & & & \\
\hline GR & 33 & 0 & 0 & 0 & 0 & 0 & 0 & 0 & 0 & 0 & 0 & 33 & 100.00 & 0.00 & 100.00 & 100.00 \\
\hline HL & 0 & 56 & 0 & 0 & 0 & 0 & 5 & 0 & 3 & 0 & 0 & 64 & 87.50 & 1.13 & 97.65 & 88.89 \\
\hline MG & 0 & 0 & 65 & 0 & 1 & 0 & 0 & 0 & 0 & 0 & 0 & 66 & 98.48 & 0.57 & 99.33 & 97.01 \\
\hline MR & 0 & 0 & 0 & 55 & 0 & 0 & 0 & 0 & 0 & 1 & 2 & 58 & 94.83 & 0.19 & 99.33 & 96.49 \\
\hline ฮี $\mathrm{PS}$ & 0 & 0 & 0 & 0 & 53 & 0 & 0 & 0 & 0 & 0 & 0 & 53 & 100.00 & 0.18 & 99.83 & 99.07 \\
\hline $\mathrm{PC}$ & 0 & 0 & 0 & 0 & 0 & 35 & 0 & 0 & 1 & 0 & 0 & 36 & 97.22 & 0.00 & 99.83 & 98.59 \\
\hline$\varangle \mathrm{TA}$ & 0 & 5 & 0 & 0 & 0 & 0 & 28 & 0 & 0 & 0 & 0 & 33 & 84.85 & 0.89 & 98.32 & 84.85 \\
\hline TI & 0 & 0 & 1 & 0 & 0 & 0 & 0 & 72 & 1 & 0 & 0 & 74 & 97.30 & 0.19 & 99.50 & 97.96 \\
\hline TS & 0 & 0 & 2 & 0 & 0 & 0 & 0 & 1 & 74 & 0 & 1 & 78 & 94.87 & 1.35 & 98.15 & 93.08 \\
\hline $\mathrm{XP}$ & 0 & 0 & 0 & 0 & 0 & 0 & 0 & 0 & 0 & 46 & 0 & 46 & 100.00 & 0.18 & 99.83 & 98.92 \\
\hline $\mathrm{ZC}$ & 0 & 1 & 0 & 1 & 0 & 0 & 0 & 0 & 2 & 0 & 50 & 54 & 92.59 & 0.55 & 98.82 & 93.46 \\
\hline Total & 33 & 62 & 68 & 56 & 54 & 35 & 33 & 73 & 81 & 47 & 53 & 595 & & & & \\
\hline
\end{tabular}

(b) Mel-Cepstrum

\begin{tabular}{|c|c|c|c|c|c|c|c|c|c|c|c|c|c|c|c|c|}
\hline & \multicolumn{11}{|c|}{ Predicted } & \multirow{2}{*}{ Total } & \multirow{2}{*}{ TP } & \multirow{2}{*}{ FP } & \multirow{2}{*}{$\mathrm{ACC}$} & \multirow{2}{*}{$\mathrm{F} 1$} \\
\hline & GR & $\mathrm{HL}$ & $\mathrm{MG}$ & $\mathrm{MR}$ & PS & $\mathrm{PC}$ & TA & TI & $\mathrm{TS}$ & $\mathrm{XP}$ & $Z C$ & & & & & \\
\hline GR & 33 & 0 & 0 & 0 & 0 & 0 & 0 & 0 & 0 & 0 & 0 & 33 & 100.00 & 0.00 & 100.00 & 100.00 \\
\hline $\mathrm{HL}$ & 0 & 59 & 0 & 0 & 0 & 0 & 5 & 0 & 0 & 0 & 0 & 64 & 92.19 & 0.94 & 98.32 & 92.19 \\
\hline MG & 0 & 0 & 66 & 0 & 0 & 0 & 0 & 0 & 0 & 0 & 0 & 66 & 100.00 & 0.00 & 100.00 & 100.00 \\
\hline MR & 0 & 0 & 0 & 58 & 0 & 0 & 0 & 0 & 0 & 0 & 0 & 58 & 100.00 & 0.19 & 99.83 & 99.15 \\
\hline สี PS & 0 & 0 & 0 & 0 & 53 & 0 & 0 & 0 & 0 & 0 & 0 & 53 & 100.00 & 0.00 & 100.00 & 100.00 \\
\hline$\exists \mathrm{PC}$ & 0 & 0 & 0 & 0 & 0 & 36 & 0 & 0 & 0 & 0 & 0 & 36 & 100.00 & 0.00 & 100.00 & 100.00 \\
\hline 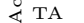 & 0 & 5 & 0 & 0 & 0 & 0 & 28 & 0 & 0 & 0 & 0 & 33 & 84.85 & 0.89 & 98.32 & 84.85 \\
\hline TI & 0 & 0 & 0 & 1 & 0 & 0 & 0 & 73 & 0 & 0 & 0 & 74 & 98.95 & 0.00 & 99.83 & 99.32 \\
\hline TS & 0 & 0 & 0 & 0 & 0 & 0 & 0 & 0 & 78 & 0 & 0 & 78 & 100.00 & 0.19 & 98.83 & 99.36 \\
\hline $\mathrm{XP}$ & 0 & 0 & 0 & 0 & 0 & 0 & 0 & 0 & 0 & 46 & 0 & 46 & 100.00 & 0.00 & 100.00 & 100.00 \\
\hline $\mathrm{ZC}$ & 0 & 0 & 0 & 0 & 0 & 0 & 0 & 0 & 1 & 0 & 53 & 54 & 98.15 & 0.00 & 99.83 & 99.07 \\
\hline Total & 33 & 64 & 66 & 59 & 53 & 36 & 33 & 73 & 79 & 46 & 53 & 595 & & & & \\
\hline
\end{tabular}

In order to obtain an overall impression of the one-against-all subproblems, the above-reported confusion matrices were summed across all the representations. As a result, the following observations can be made: In ascending order, the total number of syllables that were erroneously assigned to each class (FP) were: 24 (GR), 59 (PC), 60 (ZC), 74 (MR), 76 (TI), 82 (PS), 85 (XP), 91 (TA), 134 (MG), 148 (HL) and 151 (TS). Similarly, the number of syllables that were erroneously assigned to other classes (FN), in ascending order, were: 33 (GR), 52 (PC), 60 (XP), 70 (TI), 72 (PS), 72 (ZC), 88 (TA), 101 (MR), 140 (MG), 146 (HL) and 150 (TS). In consequence, the easiest identification corresponds to GR and the most difficult ones are HL, MG and MR. Notice also that the most frequent confusions are those between HL and TA.

\section{Discussion}

In this paper, three approaches for representing bird sounds have been analyzed: 1) feature representations, 2) dissimilarity representations for signals in the frequency domain and 3) dissimilarity representations for time-varying signal transforms. Representations - for each approach — with the highest accuracies were Mel-cepstrum representation (Table $1(\mathrm{~d})$ ), dissimilarity representation for PSDs (Table 2(b) and dissimilarity representations for time-varying Mel-cepstrum (Table 4(b) ; respectively. The last one was the representation with the overall highest total accuracy. In general, all representations have good accuracies per class; however, in this case, accuracy is not a reliable performance measure due to the unbalanced nature of the multiclass problem, i.e. the sample size of a class 
is much smaller than the combined sample size of the rest of the classes. In this case, the F1 score gives more confident results.

Mel-cepstrum and descriptive features showed a good performance in both feature and dissimilarity representations, as expected because those representations are specifically designed for audio recognition. The dissimilarity representation for PSDs, in spite of being a rather simple representation, yielded an acceptable performance with a total accuracy of $80.67 \%$. Performances of dissimilarity representations for time-varying signal transforms are remarkable. This fact reveals the importance of taking into account the non-stationarity; which is observable by comparing results of dissimilarity representations computed for FFTs, the poorest ones with a total accuracy of $50.58 \%$, and results of the dissimilarity representations for time-varying FFTs (SFTs) that had a total accuracy of $93.78 \%$. In the case of PSDs, the performance also increased when deriving dissimilarities for time-varying transforms but in less proportion, with a total accuracy of $86.22 \%$.

In summary, we conclude that Mel-cepstrum coefficients are suitable for bird sound representation, even more when dissimilarities are computed from them; i.e. when non-stationarity is taken into account. Furthemore, classifying in dissimilarity spaces derived from time-varying representations was found to be preferable instead of doing so in the corresponding 1-D representations.

Acknowledgments. This research is supported by "Programa Jóvenes Investigadores e Innovadores 2010, Convenio Interadministrativo Especial de Cooperación No. 146 de enero 24 de 2011 entre COLCIENCIAS y la Universidad Nacional de Colombia Sede Manizales" and the research program "Fortalecimiento de capacidades conjuntas para el procesamiento y análisis de información ambiental" (code Hermes-12677) funded by Universidad Nacional de Colombia.

\section{References}

1. Brenowitz, E., Margoliash, D., Nordeen, K.: An introduction to birdsong and the avian song system. Journal of Neurobiology 33(5), 495-500 (1997)

2. Acevedo, M.A., Corrada-Bravo, C.J., Corrada-Bravo, H., Villanueva-Rivera, L.J., Aide, T.M.: Automated classification of bird and amphibian calls using machine learning: A comparison of methods. Ecological Informatics 4(4), 206-214 (2009)

3. Fagerlund, S.: Bird species recognition using support vector machines. EURASIP Journal on Advances in Signal Processing 2007(1), 64-64 (2007)

4. Chou, C., Liu, P., Cai, B.: On the Studies of Syllable Segmentation and Improving MFCCs for Automatic Birdsong Recognition. In: Asia-Pacific Services Computing Conference, APSCC 2008, pp. 745-750. IEEE (2009)

5. Pękalska, E., Duin, R.P.W., Paclík, P.: Prototype selection for dissimilarity-based classifiers. Pattern Recognition 39(2), 189-208 (2006)

6. Logan, B., Salomon, A.: A music similarity function based on signal analysis. In: IEEE International Conference on Multimedia and Expo, ICME 2001, pp. 745-748 (August 2001)

7. Rubner, Y., Tomasi, C., Guibas, L.: The Earth Mover's Distance as a Metric for Image Retrieval. International Journal of Computer Vision 40(2), 99-121 (2000) 